\title{
The effects of coding procedure on human information transmission
}

JERAY G. LAMB, GENERAL DYNAMICS, ELECTRIC BOAT DIVISION HERBERT KAUFMAN, UNIVERSITY OF CONNECTICUT

Three different coding schemes, i.e., transformations between stimuli and responses, were used in a continuous information transmission situation. The stimuli were groups of binary digits and $S$ was required to transform these into verbal equivalents of combinations formed by taking various sets of the English letters. Three codes, one based on communication theory, one based on Miller's (1956) chunking hypothesis, and a combination of the first two were used. In addition, two levels of stimulus redundancy, zero and 0.5 were used. Time to complete the task and errors were the dependent variables. The Ss were run for twelve days. The basic result was that $S$ s transmitted information at a constant rate regardless of the code or redundancy level used.

Since the introduction of information theory constructs into psychology, psychologists have attempted to measure the maximum information transmission rate, or channel capacity, of the human. Previous investigators found that the ability of the human to act as a transmitter of information varies with the experimental procedure. Three general types of procedures have been used: (1) the measurement of choice reaction time (CRT) as a function of the Information in the stimulus, (2) continuously executed CRT takk, where a new stlmulus appears shortly after the response to the previous stimulus has been completed, and (3) continuous transmission tasks, such as typing, reading, etc., where sequences of stimuli are continuously arallable to $S$. These studies led to different estimates of human channel capacity for the different experimental situations. They do not answer the basic question of how the stimulusresponse code, that 1s, the transformation between stimulus and response, affects information transmission rate. The basic coding theorem of Shannon (1948) in information theory states that an optimal code exists for each communication system. To approach the maximum bit rate, channel capacity, groups of stimuli must be encoded in some manner for transmission. Miller (1956) showed that a coding procedure, which he called "chunking," strongly affected the short-term memory or information storage capacity of the human. No analogous studies have been performed on the measurement of human transmission rate.

The purpose of this study was to investigate the effects of different coding schemes and amount of stimulus information on information transmission rate in a continuous transmission situation. Three code types were used. The first was based on the chunking concept advanced by Miller; each stimulus (four digits) was coded into a single verbal response. The second was constructed to be a nearly optimal code in the Information theory sense of optimal. The final code used a combination of the first two procedures.

\section{Subjects}

\section{METHOD}

Twelve Ss, nine male and female graduate students and one faculty member at the University of Connecticut and two non-students, participated in this experiment. None had practice with the taskprior to the experiment.

\section{Stimuli}

The stimuli, shown in Fig. 1, were series of binary digits, zeros and ones, printed on standard computer paper. Each sheet consisted of a group of four digits with a two-digit space left between successive stimuli. The grouping by four of binary digits allows 16 possible stimuli. These are shown in Table 1 with associated response codes.

The stimuli were generated on an IBM 7040 computer using the random number generator avallable on the system subroutine library. Sampling was with replacement; therefore any particular stimulus could

Table 1. Stumulus Set, Probabilities Under Both Redundancy Conditions, and Associated Codes

\begin{tabular}{ccccc} 
Stimulus & & \multicolumn{2}{c}{$\begin{array}{c}\text { Response Codes (Verbal Equivalents } \\
\text { of the letters below) }\end{array}$} \\
\hline & $\begin{array}{c}(0+50) \\
\text { Chunked }\end{array}$ & $\begin{array}{c}(0+50) \\
\text { Mnemonic }\end{array}$ & $\begin{array}{c}\text { Communication } \\
(0)\end{array}$ & $\begin{array}{c}\text { Communication } \\
(50)\end{array}$ \\
\hline 0000 & A & A & AAAA & A \\
1000 & B & BA & BAAA & B AAA \\
0100 & C & BB & ABAA & B AAB \\
0010 & D & BC & AABA & B ABA \\
0001 & E & BD & AAAB & B ABB \\
1100 & F & CAB & BBAA & BB AAA \\
1010 & G & CAC & BABA & BB AAB \\
1001 & H & CAD & BAAB & BB ABA \\
0110 & I & CBC & ABBA & BB ABB \\
0101 & J & CBD & ABAB & BB BAA \\
0011 & K & CCD & AABB & BB BAB \\
0111 & L & DA & ABBB & BBB AAA \\
1011 & M & DB & BABB & BBB AAB \\
1101 & N & DC & BBAB & BBB ABA \\
1110 & 0 & DD & BBBA & BBB ABB \\
1111 & P & E & BBBB & BBBB \\
\hline
\end{tabular}


$010 \quad 0000 \quad 0000 \quad 0000 \quad 1000 \quad 0000 \quad 0010 \quad 0000 \quad 1010 \quad 0000 \quad 0000 \quad 00000001 \quad 0000 \quad 0010 \quad 0000 \quad 0000 \quad 0001 \quad 0000 \quad 0000$

\begin{tabular}{|c|c|c|c|c|c|c|c|c|c|c|c|c|c|c|c|c|c|c|c|}
\hline 0000 & 0000 & 0000 & 0000 & 0000 & 0000 & 0000 & 0010 & 0000 & 0000 & 0000 & 0000 & 0000 & 0000 & 0001 & 0000 & 0000 & 0000 & 0100 & 1001 \\
\hline 0000 & 0000 & 0010 & 0100 & 1000 & 0000 & 0000 & 0000 & 0100 & 0000 & 0001 & 0101 & 0100 & 0100 & 0010 & 1000 & 0000 & 0001 & 1000 & 0000 \\
\hline 0000 & 0000 & 1000 & 0000 & 0000 & 0000 & 0010 & 0010 & 1000 & 0000 & 1000 & 0000 & 0000 & 0100 & 0000 & 0000 & 0000 & 0001 & 0000 & 0001 \\
\hline 1001 & 0000 & 0000 & 1000 & 0000 & 0000 & 0000 & 0000 & 1000 & 0010 & 0000 & 0100 & 0000 & 0000 & 0000 & 0000 & 0000 & 1000 & 0000 & 0000 \\
\hline 0000 & 1000 & 0000 & 1100 & 0000 & 0000 & 0000 & 0000 & 0100 & 0000 & 0000 & 0000 & 0000 & 0000 & 0000 & 0000 & 0000 & 1000 & 0000 & 0000 \\
\hline 0000 & 0000 & 0000 & 0000 & 0000 & 0001 & 0000 & 0000 & 0000 & 0010 & 1000 & 0000 & 0001 & 0000 & 0000 & 0000 & 0000 & 0000 & 0000 & 0100 \\
\hline 0000 & 0001 & 0000 & 0000 & 0010 & 0010 & 0000 & 0000 & 0000 & 1001 & 0000 & 0000 & 0000 & 0100 & 0000 & 0010 & 0000 & 0000 & 0000 & 0000 \\
\hline
\end{tabular}

Fig. 1. Sample stimulus sheet for practice and transfer sessions.

occur any number of times dependent only on the input probabilities. Stimulus information was calculated for groups of 10 pages (1600 stimuli or two sessions for one $S$ ), and in every case was found to depart from the theoretical by less than $0.1 \mathrm{bit}$.

\section{Experimental Conditions}

There were six experimental conditions, three code types, chunked, mnemonic, or communication, each at two levels of stimulus redundancy. The codes were all chosen to transform the stimulus groups of four numerals into verbal responses consisting of the names of the letters of the English alphabet. These were chosen because the responses were already highly practiced, leaving only the code to be learned.

Two different levels of stimulus redundancy ( 0 and .5) were used. This was accomplished by varying the probability that any particular digit position would contain a zero. For 0 redundancy, $p(0)=p(1)=.5$; while for .5 redundancy, $p(0)=.9, p(1)=.1$. There were no sequential dependencies in either condition.

The "chunked" code was similar to one proposed by Miller (1956) to increase storage capacity in short-term memory. Each of the 16 possible messages of four digits was assigned one of the letters of the alphabet between $A$ and $P$ inclusive. This encoding. procedure chunks each stimulus group into a single letter, that is, reduces the stimuli from many units with little information per unit to a single unit with much information per unit. This code and all others are shown in Table 1 .

An optimal communication code is one constructed so that the average information per symbol on the output is maximal, and is generally constructed so that input and output alphabets are the same size. In order to approach the optimal code for the two redundancy conditions, it was necessary to construct a separate code for each. The communication code for 0 redundancy had response " $A$ " for 0 and " $B$ " for 1 and was an optimal code in the communication theory sense. The code for .5 redundancy was constructed to achieve maximum loading per symbol on the output.

The mnemonic code was intermediate between the chunked and the communication code in that it encoded the information in smaller units with more information per unit than did the communication code but to a lesser extent than the chunked code. The code was mnemonic in the sense that number and position of the input zeros and ones were directly represented in the output code, and the code was learned perfectly in a single trial by all Ss. The codes differed with respect to the size of the response alphabets; Table 2 shows the number of symbols each code used to reproduce four input symbols.

The amount of information transmitted if $\mathrm{S}$ makes 
no errors is 4.00 bits per stimulus for zero redundancy and 1.88 bits per stimulus for .5 redundancy. These values are the same for all code types since information per stimulus is invariant under the code transforms.

\section{Procedure}

Each $\mathrm{S}$ served in 12 experimental sessions. Each session lasted from 20 to $60 \mathrm{~min}$. Two Ss were assigned randomly to each group. In addition, one $S$ served an additional 50 experimental sessions, participating in all experimental conditions.

The first 10 sessions for each $S$ consisted of practice on one of the six experimental conditions. During the first session, Ss were read instructions specifying the code to be used, the stimulus probabilities, and the procedure for reading the stimuli. Each page was read aloud as from a book, that is, left to right and top to bottom. The $S$ began reading each page at E's command and read at his own rate to the bottom of the page. The amount of time necessary to read each page was measured to the nearest second with a stopwatch; $E$ also had a sheet with correct responses for each condition and scored all mistakes. The $\mathrm{E}$ told $\mathrm{S}$ his time and any mistakes made for each page. Each session consisted of reading five sheets with 160 stimuli per sheet; a sheet with S's code was available at all times. After the
Table 2. Number of Symbols in the Stimulus and Number of Symbols Necessary for Each Code to Reproduce Stimulus

\begin{tabular}{lcccc} 
Condition & \multicolumn{4}{c}{ Number of Symbols } \\
\hline $\begin{array}{l}\text { Redundancy } \\
\text { Level }\end{array}$ & Stimulus & Chunked & Mnemonic & Communication \\
\hline 0 & 4.00 & 1.00 & 2.25 & 4.00 \\
.5 & 4.00 & 1.00 & 1.39 & 2.08 \\
\hline
\end{tabular}

first session, Ss were asked at the beginning of each sesston if there were any questions about procedure.

The final two sessions each consisted of two parts. In the first part (response reading), $S$ read aloud five sheets of responses, that is, the stimull were the letters of S's code rather than binary digits (Fig. 2). The letters were printed in the same general format, and $S$ read them page by page as during the practice session. The response reading condition was included to determine if $\mathrm{Ss}$ had reached a maximum reading rate. In the other part of each of the final two sesstons, $S$ read five sheets of binary digits under the redundancy condition on which he had not practiced, and following the same procedures as the previous sessions. Appropriate instructions were given before the first test session, and errors and reading rate were measured as during the practice sessions.

\begin{tabular}{|c|c|c|c|c|c|c|c|c|c|c|c|c|c|c|c|c|c|c|c|}
\hline 1 & $c$ & $E$ & 0 & $\bullet$ & $\underline{E}$ & $p$ & c & 1 & $\boldsymbol{N}$ & $A$ & * & 2 & $\mathbf{J}$ & $c$ & 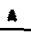 & $D$ & A & $\mathbf{K}$ & 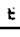 \\
\hline$\alpha$ & $\bullet$ & 6 & H & 0 & 3 & A & $k$ & c & 6 & $J$ & 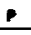 & $\mathbf{J}$ & $\boldsymbol{E}$ & 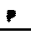 & $\boldsymbol{H}$ & I & 6 & 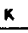 & $\mathbf{J}$ \\
\hline 4 & 1 & $\omega$ & 2 & 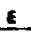 & $x$ & $n$ & $\bullet$ & n & 6 & H & 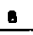 & $K$ & $\mathbf{N}$ & $\mathrm{I}$ & 4 & $M$ & $v$ & $\mathbf{I}$ & $F$ \\
\hline c & 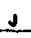 & 1 & 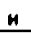 & $L$ & - & $N$ & P & K & 0 & $L$ & $L$ & c & $E$ & B & $t$ & 6 & 0 & $K$ & c \\
\hline 1 & $I$ & 1 & $F$ & \lrcorner & $\Delta$ & c & $\mathrm{k}$ & 0 & N & 0 & $\mathbf{E}$ & 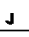 & p & 4 & 8 & $p$ & $M$ & G & $N$ \\
\hline 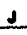 & 1 & $M$ & $L$ & 1 & $n$ & L. & $n$ & o & $n$ & \lrcorner & p & a & $E$ & 0 & 0 & B & $\vec{E}$ & $m$ & 3 \\
\hline$F$ & 6 & $c$ & \lrcorner & $p$ & N & $I$ & $c$ & A & c & E & $\mu$ & $A$ & $G$ & 4 & $\mathbf{p}$ & $\mathbf{p}$ & $\kappa$ & A & $K$ \\
\hline 0 & $k$ & $\mathbf{n}$ & 0 & $n$ & N & 1 & $\mathbf{K}$ & D & $\mathbf{L}$ & 1 & $f$ & $\mathbf{F}$ & $\mathrm{J}$ & 0 & 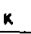 & $F$ & $\mathbf{K}$ & a & L \\
\hline
\end{tabular}

Fig. 2. Sample stimulus sheet for response reading sessions. 


\section{RESULTS ANO DISCUSSION}

The basic dependent variable was the amount of time necessary for $S$ to read aloud 160 stimull (one page). The average for the five pages of each session was used to calculate appropriate transmission rate scores. A record was kept of the number of errors; the error rate was less than one percent for all Ss for all conditions and was therefore not considered in the calculation of rates.

\section{Information Transmission Rare}

Figure 3 shows the plot of bits per sec. versus sessions for all groups. The curves for all groups except the Communication Code for .5 redundancy were so intermingled that only the envelope enclosing the curves is shown. Analyses of variance were performed on this score for the final session and over all sessions. Both analyses revealed no significant effect of either code type or redundancy, although there was a significant session (practice) effect $(F=166.73, d f=9 / 54, p<.01$ ) and a signifleant session by redundancy interaction $(F=3.45$, df $=9 / 54$, $\mathrm{p}<.01)$. Although the Communication Code for .5 redundancy group was lower than the other groups, there were no significant differences among groups. That Ss transmit information at a constant rate was the basic result of the experiment. Transmission rate was approximately five bits per sec., a result consistent with results of the CRT experiments (Hick, 1952; Hyman, 1953; Crossman, 1956; Lamb \& Kaufman, 1965; Kaufman \& Lamb, 1966). The constant information transmission rate determines the response rate (number of symbols spoken per sec.), that is, the response rate level is such that the transmission rate remains constant. This is so in spite of the fact that the group with the highest response rate must emit responses approximately five times faster than the group with the lowest response rate. The constant information rate also determines the speed at which stimuli will be read; the 50 percent redundancy groups process stimuli approximately twice as fast as the zero redundancy groups. The codes also differed, as pointed out above, in the degree to which they approximated an optimal code. In spite of these differences, all groups maintained a constant transmission rate.

The fact that $\mathrm{Ss}$ were transmitting information at a constant rate has certain implications which are noted briefly above. The first is that neither the maximal rate at which Ss can emit responses nor the rate at which $S s$ can read stimuli is the sole determinant of transmission rate. The second implication is that the efficiency, in the communication theory sense, of the codes used does not determine transmission rate. Tests were performed to test whether these implications were in fact true.

If the information load per response had little effect, as in Miller's short-term memory work, then

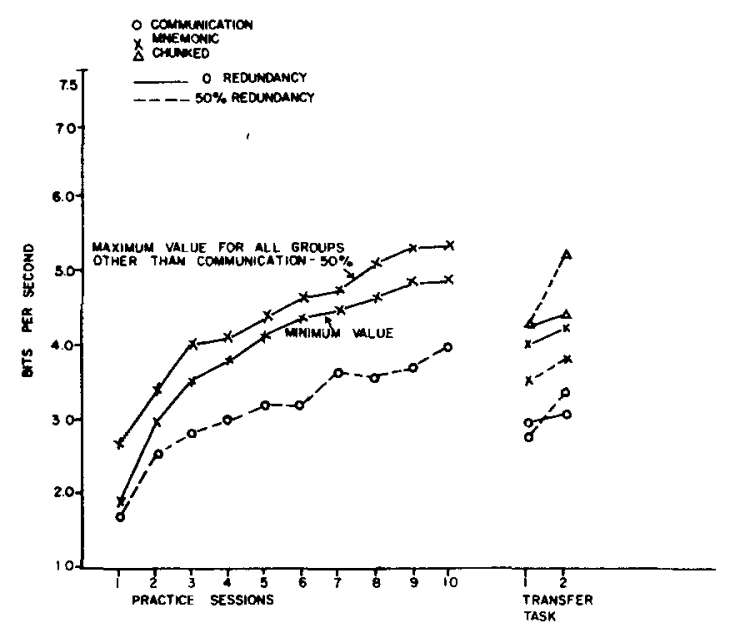

Fig. 3. Infomation transmission rates for all groups and all conditions (all groups except Communication Code for $50 \%$ redundancy are within maximum and minimum bounds).

the determining factor should be the rate at which Ss can emit responses. The response rate, number of symbols spoken per sec., was derived by calculating the average number of symbols per page for each code and dividing by the average time per page. Two analyses of variance were performed on this score. The first was on the data for the final training session. There is a significant effect due to code type $(F=38.20, d f=2 / 6, p<.01)$ and a significant interaction of code type and redundancy $(F=$ 6.09 , df $=2 / 6, p<.05$ ). The significant interaction reflects the fact that the score for the communication code and 50 percent redundancy group is below the communication code and zero redundancy group, while the scores for the other code types for 50 percent redundancy are higher than the corresponding zero redundancy groups. The second analysis was performed on the scores for all 10 practice sessions. Code type is again the largest effect $(F=52.01, \mathrm{df}=2 / 6$, $p<.005)$, although redundancy and the code type and the redundancy interaction are also significant $(\mathrm{p}<.05)$. The redundancy effect and the interaction are again the result of the communication code type being relatively lower than the other code types for the 50 percent redundancy condition. These analyses make it clear that response rate as such is not determining transmission rate under these conditions.

A variation of the first implication was that the response rate was different for the different groups because Ss were reading the stimuli at a maximum rate; 1.e., the stimulus input rate rather than response output rate was determining S's performance. Therefore analyses of variance were performed on the number of stimulus groups per sec. The results for the final practice session indicate a very large effect of redundancy $(F=292.0, d f=1 / 6, p<.001)$, and 
also a significant $(F=8.67, d f=2 / 6, p<.05)$ code type effect and a significant $(F=6.67, d f=2 / 6, p<.05$ ) interaction. The interaction is again due to the communication code for 50 percent redundancy being lower than the other code types for that redundancy. The analysis over all 10 practice sessions again shows a large effect $(F=191.26, \mathrm{df}=1 / 6, p<.001)$ due to redundancy and smaller effects due to code type and the interaction of code type and redundancy.

The results examined above support the first implication completely. Neither S's response rate (number of symbols spoken per sec.) nor S's stimulus input rate (number of stimuli read per sec.) was constant under the experimental conditions used. Miller had previously shown that encoding procedures based on the chunking concept could be used to increase the information processing capabilities of the human in a short-term memory situation. Under conditions of the present experiment, however, the degree of chunking of the S-R code had no effect on S's rate of information transmission.

The second implication was that efficiency of the code for information transmission would be the determining factor in the present situation. A standard measure of code efficiency is the number of bits in the output per symbol into the system. This is a measurement of "efficiency" in a reverse sense; the smaller the number, the more efficient the code. It is computed by calculating the average number of symbols in the output code, multiplying by the nominal Information $\left(\log _{2} M\right)$ of the output code alphabet size, and dividing by the number of input symbols that the output code represents. To conceptualize it in another fashion, the efficiency measure is the number of output bits, on the average, necessary to reproduce one bit of input. For any particular information source the lower bound on the code efficiency is the stimulus information, and if this value is reached, then the code is an optimal one.

The hypothesis was that the time rate of the code efficiency would be constant. Transmission rate (bits per sec.) would not be the same for all groups since the codes had different efficiencies, but if code efficiency is the determining factor, then the bits out per symbol in measure should be constant with respect to time. An analysis of variance was performed on the bits out per symbol in per sec. for the final practice session. Code type, rechundancy, and the interaction of code type and redundancy interaction are all significant $(p<.01)$. Contrary to the operation of theoretical communication systems, code efficiency did not determine human behavior under the conditions of this experiment.

\section{Limitations on Constant Transmission Rate}

No significant differences due to the code type or redundancy variables were found for the grouped data (Fig. 3). However, the fact that the Communica- tion Code for .5 redundancy was lower than other groups leads to the question: Is this a consistent effect? To answer this question, the data for the one $S$ who participated in all conditions (Fig. 4) was analyzed. With inter-S variability removed, there was a significant effect due to code type $(F=20.7$, $\mathrm{df}=2 / 24, \mathrm{p}<.01)$. The score of the one $\mathrm{S}$ for the communication-50 percent recundancy is lower than that of the other conditions by approximately the same amount (one bit per sec.) as for the group data. Therefore, the difference, while small relative to inter-S variability, is a consistent effect.

Stimulus input rate for this condition is lower than that for the other 50 percent redundancy conditions with the same stimuli. Since the stimuli are the same, stimulus factors cannot account for differences in information transmission rate. The response rate (number of symbols per sec.) is not as high as that of the communication code type for zero redundancy; therefore response rate is not limiting information transmission for this group. However, this is the only group which was required to reach both a high stimulus input rate (made necessary by the low Information loading of each stimulus for the 50 percent redundancy level) and a high response rate (because long response sequences of up to six letters are made necessary by the code used with an alphabet size of two). Therefore, although neither input nor output rate reached the maximum levels obtained by other groups in the present study, a simultaneous high level of both input and output may have exceeded S's capacity. It appears that mechanical limitations on S's abllity to sample and respond rather than a processing limitation are acting in this situation.

\section{Dther Resulis}

Information transmission rate as a function of practice. Figure 3 also shows that the practice function

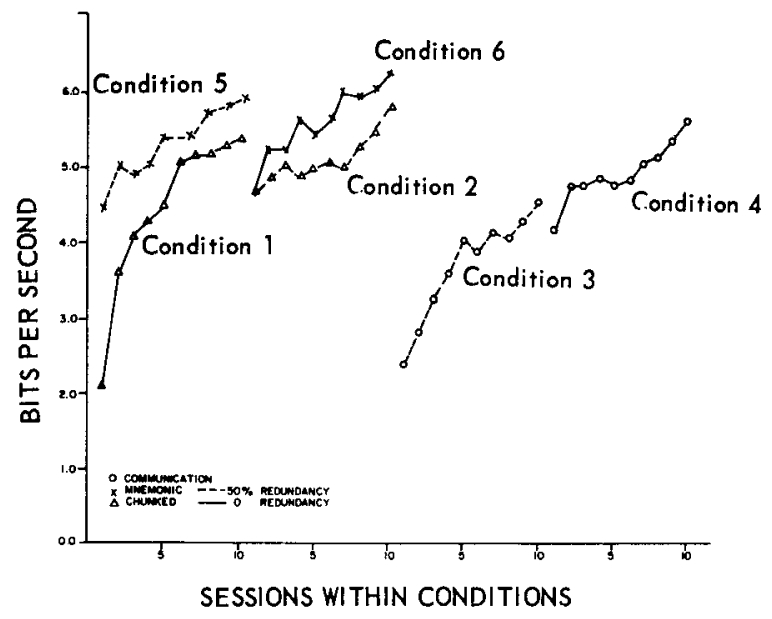

Fig. 4. Information rates for one $S$ in all conditions (Conditions are numbered in order of presentation). 
for all groups is essentially the same, that 1s, all groups reached the same final rate of information transmission at the same rate. This is a most surprising finding, since there appeared to be wide differences in the ease of learning the codes, some groups never referring to the code sheet avallable to them after the first session while other groups used it for several sessions. Another fact that supports the Idea that the rate of learning the code did not affect the rate of increase of the bits per sec. measure is that the groups who had the same code (chunked or mnemonic) but different redundancies had differential familiarity with elements of the same code. For zero redundancy, all elements appeared equally often, while for 50 percent redundancy some stimuli appeared very frequently and others very rarely. In spite of these differences in familiarity, these groups have the same bit per sec. for sessions function. As for chunking and code efficiency, the rate of information transmission seems to be independent of acquisition factors. The Ss learned to utilize the information handling capabllities of the code at a rate dependent not on the difficulty of learning the code but on the rate of information transmission.

Response reading results. During the final two sessions, Ss were required to read sheets containing the alphabetic symbols corresponding to the responses. The response reading sessions were equivalent to the practice sessions with the exception that encoding did not take place. Analyses performed on the number of symbols per sec. for the second session of response reading showed no differences among groups for symbols per sec., but significant differences in information transmission rate. These results are exactly opposite those for the practice sessions. For response reading, response rate was constant and information transmission rate varied, while for the practice sessions, information transmission rate was constant and response rate varled. The results indicate that there were no differences among the groups in ability to emit responses.

An interesting point is that Ss reached a maximum information transmission rate of approximately 14 bits/sec. for response reading with 16 letters. If the set and subset argument advanced by Fitts and Switzer (1962) is valid, then Ss would read from the entire 26-letter alphabet at about $17 \mathrm{bits} / \mathrm{sec}$.

Results on transfer task. The chunked code, contrary to the original hypothesis, was not superior to the other codes during the practice sessions. During the two transfer sessions (binary digit stimuli with other redundancy), code types had a significant effect on the information transmission rate $(F=9.40, \mathrm{df}=2 / 6, \mathrm{p}<.05)$, with the codes ranked by the degree of chunking in the codes (Fig. 3). That this superiority does not last is indicated by the data from the one $S$ who participated in all conditions (Fig. 4).

\section{DIsCussion}

Under the conditions of the present experiment, Ss transmitted information at a constant rate over a variety of code types and levels of stimulus redundancy. This constant rate for all conditions was achieved in spite of the necessity for some Ss to maintain a response rate five times that of other groups or a stimulus input rate twice that of other groups. Only in the group with both a high input and a high output rate was there any lowering of the transmission rate, and this lowering was nonsignificant for the grouped data. The efficiency, in informational terms, of the procedure used for encoding, does not determine S's transmission rate, although greater effort is required in the case of inefficient codes. It had also been hypothesized that the psychological "chunking" effect found in the work on short-term memory could be used to increase S's transmission rate. This hypothesis was clearly not substantiated. Thus under the conditions of this experiment, $S$ functions as a communication channel in a very special sense, that is, $S$ is able to transmit information at a constant rate under varying conditions.

Previous continuous transmission tasks have used complexly encoded responses such as piano playing or typing. The main purpose of the present experiment was to investigate the effect of different unfamiliar complex codes for the same input stimuli. No difference was found, indicating that the types of codes used had no effect on information transmission. Codes used in previous continuous transmission studies were very highly practiced. Skills such as oral reading have been practiced over a period of years and hundreds of thousands of responses, while in the present experiment, each $S$ made only 10,000 responses. Hellyer (1963) found that extended practice increased the rate of information transmission. The one $S$ who participated in all experimental conditions of the present experiment gradually increased his rate of transmission a total of one bit per sec. over 60 experimental sessions. Although the results show that practice can increase transmission rate, it does not seem likely that practice alone could increase the rate in the present situation to the maximum level achieved in the response reading conditions.

Another difference between the present study and previous work has to do with the stimuli which are to be encoded. In the present experiment, the stimuli were binary digits, that is, drawn from an alphabet of size two. For reading the English language, the alphabet size is 26 letters, with a consequent increase in the information for each symbol. It is 
possible that as the information loading of the input symbols increases, the rate at which $S$ can handle the input symbols is not reduced proportionately to the increase in loading and therefore information transmission rate increases. In fact, this seems to be the variable most likely to influence significantly transmission rate, and work is in progress to determine the importance of this factor.

\section{References}

Crossman, E. R. F. W. The information capacity of the human operator in symbolic and non-symbolic control processes. In Ministry of Supply Publication WR/D2/56, Information theory and the human operator. 1956.

Fitts, P., \& Switzer, G. Cognitive aspects of information processing: I. the familiarity of S-R sets and subsets. J. exp. Psychol., $1962,63,321-329$.

Hellyer, $\mathbf{S}$. Stimulus-response coding and amount of information as determinants of reaction time. J. exp. Psychol., 1963, 65, 521522.

Hick, W. E. On the rate of gain of information. Quart. J. exp. Psychol., 1952, 4, 11-26.
Hyman, R. Stimulus information as a determinant of reaction time. J. exp. Psychol., 1953, 45, 188-196.

Kaufman, H., \& Lamb, J. Choice reaction time and unequal stimuius frequencies in an absolute judgment situation. Percept. \& Psychophysics, 1966, 1, 385-387.

Lamb, J., \& Kaufman, H. Information transmission with unequally likely alternatives. Percept. mot. Skills, 1965, 21, 255-259. Miller, G. A. The magical number seven, plus or minus two: some limits on our capacity for processing information. Psychol. Rev., $1956,63,81-97$.

Shannon, C. E. Mathematical theory of communication. Bell System Tech. J., 1948, 27, 379.

\section{Note}

1. Acknowledgement is due the Office of Naval Research which in part supported this program through a prime contract (NOnr 2512 (00)) with Electric Boat Division of General Dynamics as a part of the SUBIC (Submarine Integrated Control) program, and to a predoctoral research grant from the U. S. Public Health Service. The computational part of this work was carried out in the Computer Center of the University of Connecticut, which is supported in part by Grant GP-1819 of the National Science Foundation.

(Accepted for publication February 21, 1967.) 